\title{
Volume and Shape Preservation of Enhancing Lesions when Applying Non-rigid Registration to a Time Series of Contrast Enhancing MR Breast Images
}

\author{
C. Tanner ${ }^{1}$, J.A. Schnabel ${ }^{1}$, D. Chung ${ }^{1}$, M.J. Clarkson ${ }^{1}$, D. Rueckert ${ }^{2}$, D.L.G. Hill ${ }^{1}$, \\ and D.J. Hawkes ${ }^{1}$ \\ ${ }^{1}$ Division of Radiological Sciences and Medical Engineering, \\ The Guy's, King's and St. Thomas' Schools of Medicine and Dentistry, \\ Guy's Hospital, London SE1 9RT, UK \\ 2 Department of Computing, Imperial College, London SW7 2BZ, UK
}

\begin{abstract}
In this paper we show first that a non-rigid registration algorithm used to register time-series MR images of the breast, can result in significant volume changes in the region of the enhanced lesion. Since this is physically implausible, given the short duration of the MR time series acquisition, the non-rigid registration algorithm was extended to allow the incorporation of rigid regions. In this way the registration is done in two stages. The enhanced lesions are first detected using the non-rigid registration algorithm in its original form. Secondly, the region of the enhanced lesion is set to be rigid and the new algorithm is applied to integrate this rigid region into the existing registration. By definition, volume and shape will be preserved in this rigid region. Preliminary results of applying this algorithm to 15 datasets are described.
\end{abstract}

\section{Introduction}

Breast cancer is the main cause of premature death in women. Screening programs with $\mathrm{x}$-ray mammograms have helped to improve early detection of the disease. However, they are less useful to young women for two reasons. Firstly, the amount of x-ray radiation over the years is in itself a health risk, albeit small. Secondly, the radiopaqueness of dense breast tissue makes the search for a primary tumour difficult, if not impossible [1]. Contrast enhanced magnet resonance (MR) imaging of the breast has shown promising results and is currently under investigation as a screening tool for young women at genetic risk of breast cancer in the UK [2]. In this trial, radiologists evaluate the images on the basis of a standardised protocol, which includes the analysis of Gd-DTPA uptake and washout curves as well as morphological features of suspicious regions. A vital part in this analysis is the production of a subtracted image (post-contrast minus precontrast), which in theory eliminates all unchanged structures while emphasising the enhanced regions. The shape of these enhanced regions are then analysed in an attempt to characterise disease. In practise, however, motion artifacts in the subtracted images can prevent a reliable diagnosis.

Our non-rigid registration algorithm [3] has been shown to significantly reduce the effects of movement artifacts in subtracted contrast-enhanced breast MR images [4] and thus should support the detection of enhanced lesions. Non-rigid transformations can 
generally change not only the shape but also the volume of image features [5], yet the short MR acquisition time makes volume changes during a scan sequence physically implausible. Studies suggest that breast cancer has a 15-fold increase in elastic modulus in comparison to normal fibroglandular tissue [6]. Therefore, it is reasonable to assume that tumours actually do not change shape during the study, given that little or no force is applied.

Addition of a global volume conserving constraint is physically unrealistic, given that some tissue at the periphery of the field of view may move into or out of the imaged region during the course of the acquisition. While finite element modelling can simulate the elastic tissue properties including volume preservation, it relies on a prior segmentation of the different tissue classes and is extremely computationally expensive. Local volume conservation is a possible solution but will also be computationally very expensive and difficult to implement to achieve good alignment at the skin surface and the chest wall. We therefore propose a two stage algorithm in which we first apply the nonrigid algorithm to provide good quality subtraction images in non-enhancing regions. From these subtracted images we generate a mask which will contain all MR visible enhancing tissue. We then apply a local rigid body constraint to the transformation in this region in order to preserve shape and volume, and combine this with the existing transformation. The final result can then be used for morphometric analysis.

\subsection{Related Work}

Our non-rigid registration algorithm [3] is based on finding a transformation $\mathbf{T}$ between images $A$ and $B$, which is composed of a global $3 \mathrm{D}$ transformation $\mathbf{T}_{g}$ and a local transformation $\mathbf{T}_{l}$, i.e.

$$
\mathbf{T}(\mathbf{x})=\mathbf{T}_{g}(\mathbf{x})+\mathbf{T}_{l}(\mathbf{x})
$$

where $\mathbf{T}(\mathbf{x})$ transforms the position $\mathbf{x}=\left(x_{1} x_{2} x_{3}\right)^{T}$ to $\mathbf{x}^{\prime}$, which maps the point in the image $A(\mathbf{x})$ into its corresponding point in image $B\left(\mathbf{x}^{\prime}\right) . \mathbf{T}_{g}$ is given by the $3 \mathrm{D}$ rigid transformation:

$$
\mathbf{T}_{g}(\mathbf{x})=\left(\begin{array}{lll}
\alpha_{11} & \alpha_{12} & \alpha_{13} \\
\alpha_{21} & \alpha_{22} & \alpha_{23} \\
\alpha_{31} & \alpha_{32} & \alpha_{33}
\end{array}\right)\left(\begin{array}{l}
x_{1} \\
x_{2} \\
x_{3}
\end{array}\right)+\left(\begin{array}{l}
\alpha_{14} \\
\alpha_{24} \\
\alpha_{34}
\end{array}\right)
$$

where the coefficients $\alpha$ are parameterised by the 6 (or 12) degrees of freedom of a $3 \mathrm{D}$ rigid (or affine) transformation. The local transformation $\mathbf{T}_{l}$ is described by a freeform deformation (FFD) model based on B-splines, which are computationally more efficient than thin-plate splines used in [7]. The object is deformed by manipulating an underlying mesh of control points, yielding a smooth $C^{2}$ continuous transformation.

Let $\Omega=\left\{\mathbf{x} \mid 0 \leq x_{i}<P_{i}, i \in\{1,2,3\}\right\}$ be the domain of the image volume and let $\Psi=$ $\left\{\psi_{j_{1}, j_{2}, j_{3}} \mid j_{i} \in\left[0, N_{i}-1\right], i \in\{1,2,3\}\right\}$ be the mesh of control points with displacements $\phi_{j_{1}, j_{2}, j_{3}}^{(i)}$ and spacing $d_{i}=\frac{P_{i}}{N_{i}-1}$ for $i \in\{1,2,3\} . \mathbf{T}_{l}(\mathbf{x})=\left(T_{l}^{(1)} T_{l}^{(2)} T_{l}^{(3)}\right)^{T}$ can then be written as:

$$
T_{l}^{(i)}(\mathbf{x})=\sum_{l=0}^{3} \sum_{m=0}^{3} \sum_{n=0}^{3} B_{l}\left(u_{1}\right) B_{m}\left(u_{2}\right) B_{n}\left(u_{3}\right) \phi_{j_{1}+l, j_{2}+m, j_{3}+n}^{(i)} \quad i \in\{1,2,3\}
$$


where $j_{i}=\left\lfloor\frac{x_{i}}{d_{i}}\right\rfloor-1, u_{i}=\frac{x_{i}}{d_{i}}-\left\lfloor\frac{x_{i}}{d_{i}}\right\rfloor$ for $i \in\{1,2,3\}$ and $B_{l}$ is the $l$-th basis function of the B-spline defined by:

$$
\begin{array}{ll}
B_{0}(u)=(1-u)^{3} / 6 & B_{2}(u)=\left(-3 u^{3}+3 u^{2}+3 u+1\right) / 6 \\
B_{1}(u)=\left(3 u^{3}-6 u^{2}+4\right) / 6 & B_{3}(u)=u^{3} / 6
\end{array}
$$

An optimal transformation $\mathbf{T}$ is found by maximising the similarity measure between the overlapping regions of image $A$ and the transformed image of $B$, i.e $S(A, \mathbf{T}(B))$ by applying a gradient ascent technique. The normalised mutual information (NMI) was chosen as similarity measure, because the contrast enhancement prohibits direct comparison of the intensities, and any dependency on the amount of image overlap is avoided [8], i.e.

$$
S(A, B)=\frac{H(A)+H(B)}{H(A, B)}
$$

with $H(A)$ being the marginal entropy of image $A$ and $H(A, B)$ being the joint entropy of the images $A$ and $B$ calculated from their joint histogram.

\subsection{Objectives}

It was shown that non-rigid registration facilitates the detection of enhancing regions [4]. In this paper we investigate to what extent this algorithm changes the volume of an enhanced lesion. This is important since we know that tumours do not shrink or expand during enhancement, and volume changes will produce artifactual changes in the shape of lesions which may hinder attempts to characterise benign and malignant disease. We propose a method to adopt the algorithm to preserve lesion shape and volume.

\section{Experiment Design}

First we describe our method for assessing volume change. Then we describe an experiment to investigate the effect of control point spacing of the FFD algorithm. In section 2.4 we propose a novel method for conserving volume and shape of enhanced breast lesions.

\subsection{Evaluation of Volume Change}

First of all, position and extent of the enhancing lesion has to be determined as our ground truth. This has to be based on subtracted contrast enhancing MR images, since malignancies cannot be reliably detected by plain MR imaging [1]. As shown in [4], enhanced lesions can be significantly better detected when non-rigid registration was applied. Thus, our ground truth was determined in accordance with [4] by the following procedure:

1) To reduce motion artifacts, the pre-contrast image (I1) was registered to the postcontrast image obtained two minutes after injection (I2), resulting in the transformation $\mathbf{T}_{1,2}$.

2) Transformation $\mathbf{T}_{1,2}$ was then used to deform $I 1$ to $I 1^{\prime}$ to match $I 2$ using tri-linear interpolation. 
3) The transformed pre-contrast image $I 1^{\prime}$ was then subtracted from the post-contrast image $I 2$ to visualise the region of enhancements.

4) From the subtracted image $I 1^{\prime}-I 2$, the enhancing lesion was segmented using ANALYZE (Biomedical Imaging Resource, Mayo Foundation, Rochester, MN, USA). A mask image $M$ was obtained with intensity values $U$ within the enhancing lesion $L$ and 0 otherwise.

From our ground truth, the volume change induced by each of the tested registrations is evaluated by the following steps:

a) The post-contrast image $I 2$ is registered to the pre-contrast image $I 1$, resulting in the transformation $\mathbf{T}_{2,1}$.

b) The actual volume change was then determined by transforming the mask image $M$ to $M^{\prime}$ by $\mathbf{T}_{2,1}$. Tri-linear interpolation was used to transform $M$ to $M^{\prime}$, so boundary voxels in the transformed image $M^{\prime}$ will have intensities between 0 and $U$. Correcting for such partial volume effects, the volume of $M^{\prime}$ is given by

$$
V_{M^{\prime}}=\frac{V_{\text {voxel }\left(M^{\prime}\right)}}{U} \sum_{\mathbf{i} \in M^{\prime}} M^{\prime}(\mathbf{i})
$$

where $V_{\text {voxel }}\left(M^{\prime}\right)$ is the volume of a voxel in $M^{\prime}$ and $M^{\prime}(\mathbf{i})$ is the intensity of voxel $\mathrm{i}$ in $M^{\prime}$. The volume change in percent is then

$$
\Delta V=100 \times \frac{V_{M^{\prime}}-V_{M}}{V_{M}} .
$$

The dependence of the volume change on our ground truth is investigated in section 3.3. Note that $\mathbf{T}$ is in general not invertible since folding leads to violation of the one-toone mapping property. The ground truth was therefore determined by using $\mathbf{T}_{\mathbf{1 , 2}}$ and not the inverse of $\mathbf{T}_{\mathbf{2}, \mathbf{1}}$. For the registrations in accordance with [4], the average of the magnitude of the displacement error over all voxel positions in $I 1$ is $0.98 \mathrm{~mm}$ and in the lesion $L$ is $0.81 \mathrm{~mm}$. The resultant displacement vector over all voxel positions in $I 1$ is $(-0.02 \mathrm{~mm} 0.20 \mathrm{~mm} 0.08 \mathrm{~mm})^{T}$ and in $L$ is $(-0.17 \mathrm{~mm}-0.19 \mathrm{~mm}-0.03 \mathrm{~mm})^{T}$.

\subsection{Data}

The MR scans were acquired with a Siemens $1.5 \mathrm{~T}$ Impact MR system using a fast gradient echo 3D sequence with $\mathrm{TR}=12 \mathrm{~ms}, \mathrm{TE}=5 \mathrm{~ms}$, flip angle $=35^{\circ}, \mathrm{FOV}=350 \mathrm{~mm}$ and axial slice orientation. Five post-contrast scans were obtained after injection of 0.2 mmol Gd-DTPA $/ \mathrm{kg}$ of body weight at temporal intervals of 1 minute and a voxel size of $1.37 \times 1.37 \times 4.2 \mathrm{~mm}^{3}$.

For each registration method, the volume change was evaluated on 15 pairs of pre- and post-contrast images selected from the 54 pairs of pre- and post-contrast breast images used in [4]. Datasets were selected that encompassed a wide range of lesion shapes and sizes. 


\subsection{Variation of Control Point Spacing}

The spacing of the control points determines the flexibility of the non-rigid registration. In [9], the registration results for control point spacing of 20, 15 and $10 \mathrm{~mm}$ were compared in terms of the squared sum of intensity differences (SSD) and correlation coefficient (CC) of the intensities of the image pair after registration. This showed that decreasing the control point spacing reduced the registration error (excluding the tumour region).

In this paper we investigate the effects on the volume change of the lesion for a control point spacing of $10,15,20$ and $25 \mathrm{~mm}$.

\subsection{Coupling of Control Points}

The main idea of restricting control points to have the same displacement (i.e. they are coupled) is to produce regions which are only allowed to transform rigidly within the FFD. When control points are coupled, then any region, whose displacement is only controlled by these control points, will have no volume change. To be precise, let an image region $R \subseteq I 1$, be under the influence of coupled control points, i.e. for $\mathbf{x} \in R$, $\phi_{j_{1}+l, j_{2}+m, j_{3}+n}^{(i)}=K^{(i)}$, a constant, for $l, m, n \in\{0,1,2,3\}$ and $i \in\{1,2,3\}$. Then (3) simplifies to

$$
T_{l}^{(i)}(\mathbf{x})=K^{(i)} \underbrace{\sum_{l=0}^{3} \sum_{m=0}^{3} \sum_{n=0}^{3} B_{l}\left(u_{1}\right) B_{m}\left(u_{2}\right) B_{n}\left(u_{3}\right)}_{1}=K^{(i)}, \quad i \in\{1,2,3\} .
$$

Thus, the local transformation $\mathbf{T}_{l}(\mathbf{x})$ for $\mathbf{x} \in R$ is given by the translation vector $\left(K^{(1)} K^{(2)} K^{(3)}\right)^{T}$, which by definition does not introduce shape or volume changes in $R$.

Let $\Psi_{\text {coup }}$ be the set of coupled control points and $\Psi_{\text {blend }}$ be the set of control points updated for blending the coupled region into the surrounding. Then the coupling of control points can be incorporated in the registration in following way:

- find $\mathbf{T}(\mathbf{x})$ as in [4] to detect an enhancing lesion $L$

- determine bounding box $D=[\mathbf{v}, \mathbf{w}]$ of $L$ such that $L \in D$ and D has minimal volume

- include in $\Psi_{\text {coup }}$ the adjacent two control points in each direction of $D$, i.e. $\Psi_{\text {coup }}=\left\{\psi_{j_{1}, j_{2}, j_{3}} \mid j_{i} \in\left[\max \left(0,\left\lfloor\frac{v_{i}}{d_{i}}\right\rfloor-1\right), \min \left(N_{i}-1,\left\lceil\frac{w_{i}}{d_{i}}\right\rceil+1\right)\right], i \in\{1,2,3\}\right\}$

- include in $\Psi_{\text {blend }}$ the adjacent two control points in each direction of $\Psi_{\text {coup }}$, i.e. $\Psi_{\text {blend }}=$

$\left\{\psi_{j_{1}, j_{2}, j_{3}} \mid j_{i} \in\left[\max \left(0,\left\lfloor\frac{v_{i}}{d_{i}}\right\rfloor-3\right), \min \left(N_{i}-1,\left\lceil\frac{w_{i}}{d_{i}}\right\rceil+3\right)\right], i \in\{1,2,3\}\right\}-\Psi_{\text {coup }}$

- the coupled control point displacements $\Phi_{\text {coup }}$ are initialised to their mean

- using gradient asscent, the similarity measure (4) is maximised with respect to $\Psi_{\text {coup }}$ and $\Psi_{\text {blend }}$, while enforcing the displacement vectors $\Phi_{\text {coup }}$ to be the same.

This approach only allows a coupled translation. This should be a sufficient approximation for the local transformation of a rigid body, given that the global rotations are covered by $\mathbf{T}_{g}$. In further work we will investigate whether a local rigid registration (similar to [10]) combined with a FFD improves the registration. Figure 1 shows a 2D example of coupling control points to prevent shape and area change locally. 


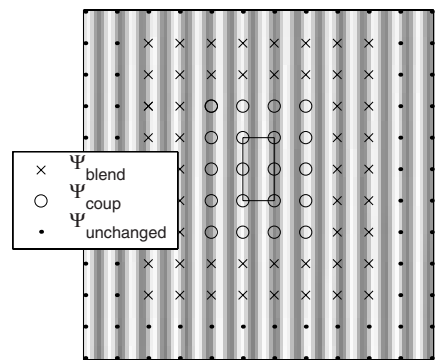

(a)

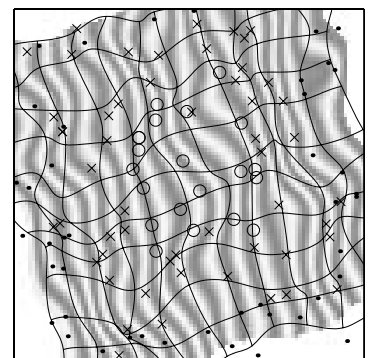

(b)

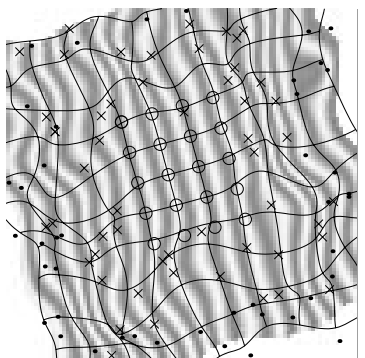

(c)

Fig. 1. 2D example of control point coupling in order to prevent shape and area change within the box depicted in (a). The global transformation consists of a $15^{\circ}$ rotation. The FFD is based on a control point spacing of 9 pixels. The control point displacements are generated randomly from a normal distribution with a standard deviation of 5 pixels. The deformed grids in (b) and (c) show the transformed position of a mesh drawn through the control points in (a). Note that the deformed grids do not necessarily pass through the displaced control points since the FFD is interpolated using B-spline approximators. (a) sinusoidal source image and the initial distribution of control points, (b) the transformed source image using bi-linear interpolation and the deformed grid and (c) the transformed source image and deformation after coupling the control points $\Psi_{\text {coup }}$. In (c), the image within the corresponding region to the marked box in (a) has not changed shape, while this is not true for (b).

\section{Results}

In this section we present results of our investigation of the control point spacing in the FFD and of our novel approach to volume and shape preservation. Finally, the dependency of the volume change on the initial lesion segmentation is evaluated.

\subsection{Variation of Control Point Spacing}

Test were conducted using our original non-rigid registration algorithm with a control point spacing of 10, 15, 20 and $25 \mathrm{~mm}$. The summarised results are given in Table 1 and are depicted in Figure 2. Volume change is measured as a percentage relative to the initial volume as defined by (6). The mean volume change over the 15 datasets lie within $\pm 0.3 \%$ for all tests with global rigid registration, indicating no global bias towards expansion or contraction. However, volume changes occur within a range of $[-17 \%, 33 \%]$ for the non-rigid registration configuration from [4], i.e. a FFD of $10 \mathrm{~mm}$ after a global affine registration. The standard deviation of the volume change increases as the FFD node spacing decreases from $25 \mathrm{~mm}$ down to $10 \mathrm{~mm}$. This indicates lager changes in volume the smaller the node spacing of the FFD. The trend of the similarity measures shows that at finer node spacing the two images are more similar. The values of the similarity measures are tabulated as negative squared sum of intensity difference (-SSD), correlation coefficient (CC) and normalised mutual information (NMI). This trend is in concordance with the results from [9] and can be explained by the reduced 
flexibility of the non-rigid registration due to a lower number of control points. The plot in Figure 2 shows that the volume changes induced by the algorithm are not correlated with lesion size.

\begin{tabular}{|c||r|r|r|r||r|r|r|}
\hline \multirow{2}{*}{\multicolumn{1}{|c||}{$\begin{array}{c}\text { Registration } \\
\text { Method }\end{array}$}} & \multicolumn{3}{c||}{ Volume Change in \% } & \multicolumn{3}{c|}{ Mean Similarity } \\
\cline { 2 - 8 } & Min & Max & Mean & \multicolumn{1}{c|}{ Std } & -SSD & CC & NMI \\
\hline \hline affine + FFD 10mm & -16.83 & 32.05 & 1.2919 & 12.0648 & -2146.46 & 0.9847 & 1.2018 \\
\hline rigid + FFD 10mm & -18.91 & 17.59 & 0.1636 & 10.2618 & -2162.61 & 0.9845 & 1.2018 \\
\hline rigid + FFD 15mm & -5.77 & 4.19 & 0.0072 & 3.2029 & -2430.76 & 0.9823 & 1.1985 \\
\hline rigid + FFD 20mm & -2.66 & 7.29 & 0.2315 & 2.3736 & -2555.84 & 0.9812 & 1.1965 \\
\hline rigid + FFD 25mm & -2.86 & 1.57 & -0.2301 & 1.4394 & -2623.89 & 0.9804 & 1.1954 \\
\hline
\end{tabular}

Table 1. Results for the original non-rigid registration algorithm with a control point spacing of $25,20,15$ and $10 \mathrm{~mm}$ after global affine or rigid registration. The volume change as well as the similarity between the two images (measured excluding the enhanced lesion) decreases with the increase of node spacing. The evaluated similarity measures are negative squared sum of intensity difference (-SSD), correlation coefficient (CC) and normalised mutual information (NMI).

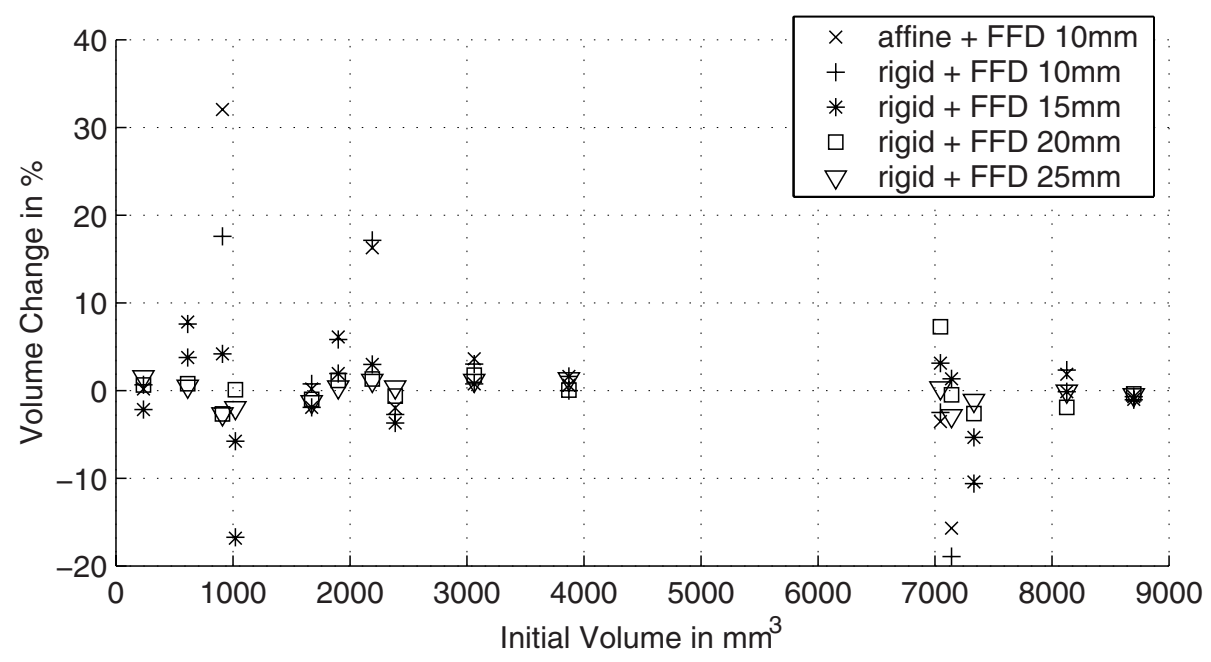

Fig. 2. The volume changes for the 15 lesions and the different methods applied. There is no correlation between initial volume and volume change.

\subsection{Coupling of Control Points}

The result of the non-rigid registration with control point spacing of $10 \mathrm{~mm}$ was chosen as the initial transformation, due to its ability to significantly reduce the effects of motion artifacts [4]. This transformation was then optimised for shape and volume preservation of the lesion while blending the lesion region with its surrounding by having two 
sets of control points, $\Psi_{\text {coup }}$ and $\Psi_{\text {blend }}$ respectively. As described in Section 2.4, the control points from $\Psi_{\text {coup }}$ lie in a dilated box bounding the lesion and are restricted to have the same displacement vectors. $\Psi_{\text {blend }}$ covers the zone that is influenced by $\Psi_{\text {coup }}$ but which is not in $\Psi_{\text {coup }}$. The results of using coupled control points are shown in Table 2 and Figure 3 in comparison to the global rigid and affine registration. Clearly, the coupling of control points is as good as the global rigid registration in preventing volume changes within the lesion. The observed volume changes indicate the measurement errors, since the rigid transformation is by definition volume preserving. Global affine transformation causes small volume changes, but improves the registration insignificantly in comparison to global rigid registration as shown in [4]. It also leads to higher volume changes when applied before the FFD with $10 \mathrm{~mm}$ and is therefore not recommended for registrations where volume should be preserved. The mean similarity achieved by coupled control points indicate a registration error similar to a global rigid registration followed by a non-rigid registration with $15 \mathrm{~mm}$ control point spacing. However, the true registration quality needs to be evaluated in an observer study. The effects of the different registrations on one lesion can be seen in Figure 5.

\begin{tabular}{|c||c|c|c|c||c|c|c|}
\hline \multicolumn{1}{|c||}{$\begin{array}{c}\text { Registration } \\
\text { Method }\end{array}$} & \multicolumn{3}{c||}{ Volume Change in \% } & \multicolumn{3}{c|}{ Mean Similarity } \\
\cline { 2 - 8 } & Min & Max & Mean & Std & -SSD & CC & NMI \\
\hline \hline coupled & -0.03 & 0.03 & 0.0016 & 0.0155 & -2400.42 & 0.9825 & 1.1989 \\
\hline rigid & -0.11 & 0.03 & -0.0039 & 0.0340 & -3469.28 & 0.9725 & 1.1895 \\
\hline affine & -0.61 & 1.19 & 0.0842 & 0.5230 & -3106.54 & 0.9759 & 1.1904 \\
\hline
\end{tabular}

Table 2. Results when the control points around the enhanced lesion are coupled in comparison to the global rigid and affine registration. The coupled control points preserve volume within the lesion as good as rigid registration while the mean similarity is within the range of rigid + FFD $15 \mathrm{~mm}$.

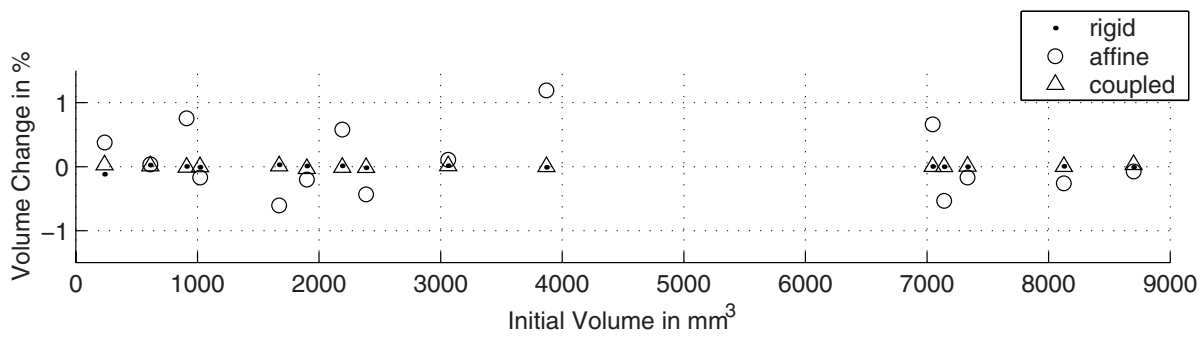

Fig. 3. The volume change of the 15 lesions for global affine and rigid registration and non-rigid registration with coupled control points within the extended region of the lesion. 


\subsection{Robustness of Results}

To analyse the dependency of the previous results on the exact segmentation of the lesions, tests were performed with dilated lesion masks in order to obtain volume change profiles similar to [11]. The mask $M$ was blurred with Gaussian filters with standard deviation of $0.2,0.4, \ldots 1.8$. Then, all intensities greater than zero were set to $U$. Finally, the dilated masks were transformed using the registrations results from before without changing the sets of coupled and blending control points. Figure 4 shows that for a 5 -fold increase of lesion volume, the standard deviation of volume change induced by the algorithm is almost constant for the FFD with node spacings of $15 \mathrm{~mm}$ and above. There is a small, but significant, reduction in volume change of between 4 and 5\% from a FFD of $10 \mathrm{~mm}$.

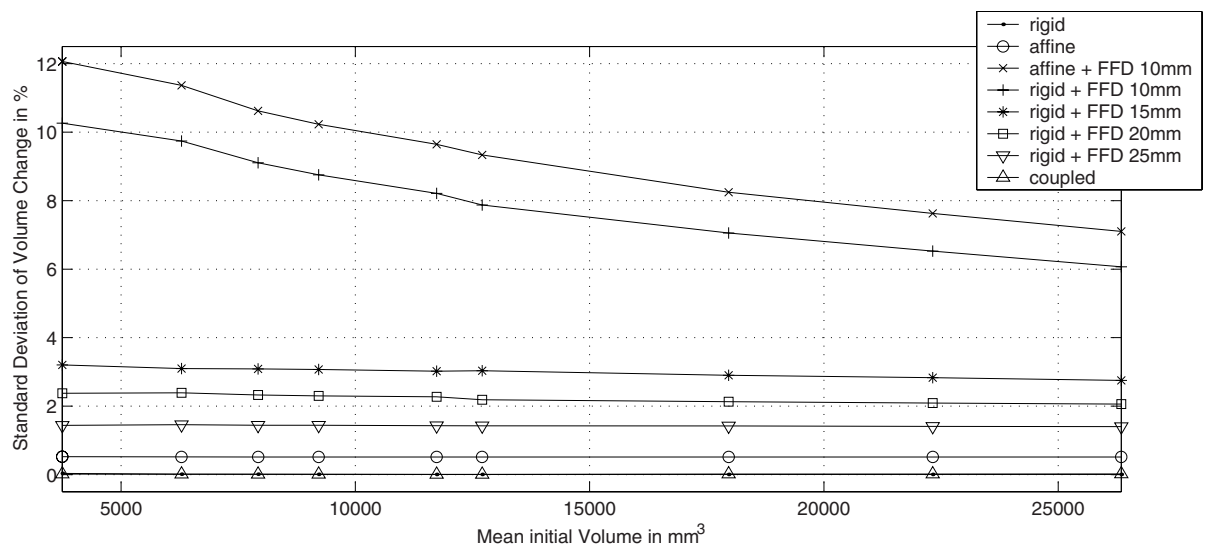

Fig. 4. Dependency of the volume change on the lesion segmentation. The segmented lesion is dilated using Gaussian blurring with a standard deviation of $0,0.2, \ldots, 1.8$ and all intensities greater than zero are set to belong to the lesion. Although the mean initial volume increases 5-folded, the volume change for these new segmentations show clearly the same ranking for the different registration methods. This indicates a weak dependency of the volume change on the lesion's segmentation.

\section{Conclusions}

In this paper we have shown that the non-rigid registration algorithm assessed in [4] can lead to significant local volume changes. We presented initial results showing that these volume changes are more severe the finer the node spacing in the FFD algorithm that we use.

We proposed a strategy in which a non-rigid registration with $10 \mathrm{~mm}$ control point spacing is first undertaken. This reduces subtraction artifacts in non-enhancing regions significantly [4] . From the subtracted image the local transformation for the region of 
the enhancing lesion is then constrained to a translation only. The transformation from the initial non-rigid registration is then optimised to account for this constraint. The final result guarantees volume and shape preservation in the region of the enhanced lesion, within the limits of the interpolation method used in the transformation, while still showing the lesion in the context of its surrounding tissue. In future work, we will extend and improve the proposed method of volume and shape preserving constraints within the framework of a generalised FFD, investigate the rigidity assumption of lesions in the context of contrast enhanced MR breast imaging and undertake an observer study to assess the effect of volume and shape preservation constraints on the quality of the subtracted images. The resulting enhancing region can then be passed on for further morphological analysis in attempt to better distinguish benign from malignant disease.
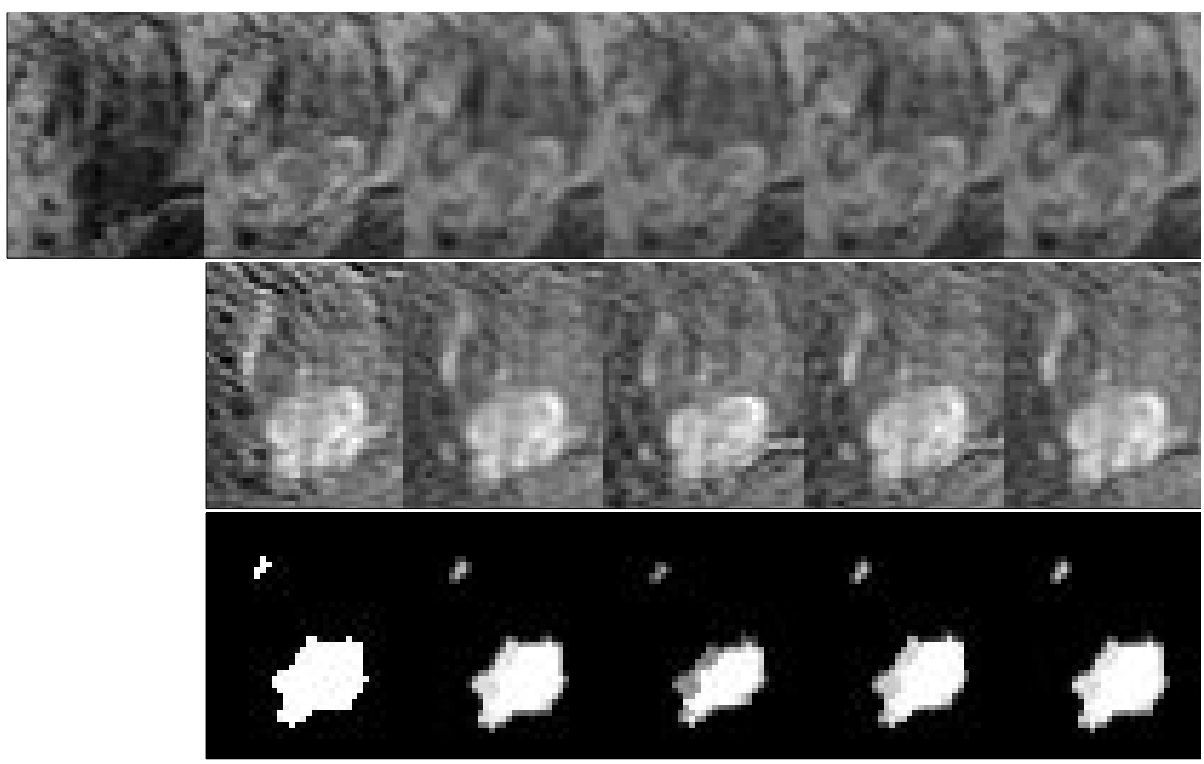

(a)

(b)

(c)

(d)

(e)

(f)

Fig. 5. This figure shows the results of registering the post-contrast image to the pre-contrast image of one patient in 4 different ways. The images display the region of the enhanced lesion on one slice. The top row shows (a) the pre-contrast image, (b) the post-contrast image and the transformed post-contrast images after (c) rigid registration, (d) non-rigid registration with a control point spacing of $10 \mathrm{~mm}$ as in [4], (e) non-rigid registration with a control point spacing of $25 \mathrm{~mm}$ after a global rigid registration and (f) non-rigid registration with coupled control points within an extended region of the lesion. The middle row shows the difference images after subtracting the pre-contrast image from the images in the top row. The bottom row pictures the initial and the transformed masks. A clear shrinkage and shape change for the transformed mask of (d) can be observed. The volume changes are (c): $0.00 \%$ (d): $-16.28 \%$ (e): $-3.86 \%$ (f): $0.00 \%$. 


\section{Acknowledgments}

We would like to thank the Engineering and Physical Sciences Research Council (EPSRC) for the financial support given by the EPSRC grant GR/M52779 and GR/L08519 and Dr. Sheila Rankin, Dr. Erika Denton and the Radiographers at Guy's and St. Thomas NHS trust for assistance in collection of the data. This work is part of a joint project with the Institude of Cancer Research and we are very grateful to Prof. Martin Leach, Dr. Carmel Hayes and Dr. Andreas Degenhard for useful discussions

\section{References}

[1] S. Heywang-Köbrunner and R. Beck, Contrast-Enhanced MRI of the Breast. Berlin, Germany: Springer-Verlag, 2nd ed., 1995.

[2] M. O. Leach, "Assessing Contrast Enhanced MRI as a Method of Screening Women at Genetic Risk of Breast Cancer: Study Design, Methodology and Analysis," in Proceedings of the Sixth Scientific Meeting of the International Society for MR in Medicine, Sydney, Australia, p. 226, 1998.

[3] D. Rueckert, L. I. Sonoda, C. Hayes, D. L. Hill, M. O. Leach, and D. J. Hawkes, "Nonrigid Registration using Free-Form Deformation: Application to Breast MR Images," IEEE Transactions on Medical Imaging, vol. 7, pp. 1-10, August 1999.

[4] E. R. E. Denton, L. I. Sonoda, D. Rueckert, S. C. Rankin, C. Hayes, M. O. Leach, and D. J. Hawkes, "Comparison and Evaluation of Rigid, Affine, and Nonrigid Registration of Breast MR Images," Journal of Computer Assisted Tomography, vol. 5, pp. 800-805, May 1999.

[5] E. A. Stamatakis, J. T. L. Wilson, and D. J. Wyper, "The Effect of Non-Linear Image Registration on Cerebral Lesions," in Medical Image Understanding and Analysis, Oxford, UK, pp. 21-24, 1999.

[6] A. Sarvazyan, D. Goukassian, E. Maevsky, and G. Oranskaja, "Elastic Imaging as a new Modality of Medical Imaging for Cancer Detection," in Proceedings of the International Workshop on Interaction of Ultrasound with Biological Media, Valenciennes, France, pp. 69-81, 1994.

[7] C. R. Meyer, J. L. Boes, B. Kim, P. H. Bland, K. R. Zasadny, P. V. Kison, K. Koral, K. A. Frey, and R. L. Wahl, "Demonstration of Accuracy and Clinical Versatility of Mutual Information for Automatic Multimodality Image Fusion using Affine and Thin-plate Spline Warped Geometric Deformations," Medical Image Analysis, vol. 1, no. 3, pp. 195-207, 1997.

[8] C. Studholme, D. L. G. Hill, and D. J. Hawkes, “An Overlap Invariant Entropy Measure of 3D Medical Image Alignment,” Pattern Recognition, vol. 32, pp. 71-86, 1999.

[9] D. Rueckert, C. Hayes, C. Studholme, P. Summers, M. Leach, and D. J. Hawkes, "Non-rigid Registration of Breast MR Images using Mutual Information," in Medical Image Computing and Computer-Assisted Intervention, Cambridge, USA, pp. 1144-1152, 1998.

[10] J. Little, D. L. G. Hill, and D. J. Hawkes, "Deformations Incorporating Rigid Structures," Computer Vision and Image Understanding, vol. 66, pp. 223-232, 1997.

[11] J.-P. Thirion and G. Calmon, "Deformation Analysis to Detect and Quantify Active Lesions in 3D Medical Image Sequences," INRIA Report, vol. 3101, February 1997. 